\title{
Crossover between multipole Coulomb and Kubas interactions in hydrogen adsorption on metal-graphene complexes
}

\author{
Gyubong Kim, ${ }^{1}$ Seung-Hoon Jhi, ${ }^{1, *}$ Seokho Lim, ${ }^{2}$ and Noejung Park ${ }^{2}$ \\ ${ }^{1}$ Department of Physics, Pohang University of Science and Technology, Pohang 790-784, Korea \\ ${ }^{2}$ Department of Applied Physics, Dankook University, 126 Jukjeon-dong, Yongin-si, Gyeonggi-do 448-701, Korea
}

(Received 15 March 2009; published 23 April 2009)

\begin{abstract}
The hydrogen adsorption on alkaline-earth metal dispersed in doped graphenes was studied through ab initio calculations. Substitutional doping in graphenes is explored to control the ionic state of the metal atoms that plays a crucial role for dispersion and hydrogen adsorption. It was found that the adsorption behavior, particularly in Ca-dispersed graphene complexes, exhibits a crossover between the multipole Coulomb and Kubastype (or orbital) interactions as the ionic state of $\mathrm{Ca}$ and the number of adsorbed hydrogen molecules change. The level exchange in $s$ and $d$ orbitals of $\mathrm{Ca}$ is responsible for the crossover. This finding enables the optimization of hydrogen adsorption and metal dispersion in graphitic materials, which is useful for developing solid hydrogen storage and efficient catalysts.
\end{abstract}

DOI: $10.1103 /$ PhysRevB.79.155437

PACS number(s): 68.43.Bc, 68.55.Ln, 73.20.Hb

The interaction of hydrogen molecules with metal atoms is a key physicochemical process involved in many energy related technology such as fuel cell catalysts and hydrogen storage. For example, developing storage systems of hydrogen in nondissociative forms has been a great challenge in hydrogen-based fuel cell research. ${ }^{1-4}$ Transition metal (TM) complexes have been studied extensively in this respect as they were shown to be very promising in terms of hydrogen binding strength and storage capacity at ambient conditions. ${ }^{5-10}$ Quantum mechanical simulations showed that the hydrogen binding energy is about $0.3-0.7 \mathrm{eV}$ depending on TM and the storage capacity can reach as high as 6 wt \%. ${ }^{5-10}$ The Kubas-type (or orbital) interaction between $\mathrm{H}_{2}$ and TM is known to be responsible for such a large number of $\mathrm{H}_{2}$ adsorbed on TM with significant binding energies. ${ }^{11}$ While many theoretical studies proved the strong potential of TM dispersion, experimental evidence has not been positive. The metal clustering was pointed out as a probable source for degradation of TM complexes. ${ }^{12,13}$ Adsorption or catalytic functionality of metal atoms with hydrogen so far has been directly associated with metal $d$ orbitals. Nontransition metals such as $\mathrm{Li}, \mathrm{Na}, \mathrm{Mg}, \mathrm{Al}$, and $\mathrm{Ca}$ usually form hydrides, where $\mathrm{H}_{2}$ dissociate to form chemical bonding with metal atoms. ${ }^{1,2}$ Lochan and Head-Gordon ${ }^{14}$ reported interesting theoretical results, which show that ionic alkalimetal atoms such as $\mathrm{Li}^{+}, \mathrm{Na}^{+}, \mathrm{Mg}^{2+}$, and $\mathrm{Al}^{3+}$ can hold up to six hydrogen molecules. This indicates that, once alkalimetal or alkaline-earth metal (AEM) exists as bare ion, it can be a binding center for multiple $\mathrm{H}_{2}$ without dissociation. As the binding energy of metal atoms to substrate depends on their ionic state, the metal dispersion can also be affected by their charge state. While many studies have dealt with metaldispersed medium for hydrogen storage, ${ }^{5-10}$ the detailed mechanism and its practical implication are not comprehensively addressed yet. Here we study the dispersion of non- $d$ metal atoms, such as $\mathrm{Be}, \mathrm{Mg}, \mathrm{Al}$, and $\mathrm{Ca}$, in doped graphenes and the hydrogen adsorption on these metals with the use of $a b$ initio methods including the density-functional theory (DFT) and the Møller-Plesset second-order perturbation method (MP2). ${ }^{15}$ The adsorption property of hydrogen mol- ecules is particularly studied for $\mathrm{Mg}$ and $\mathrm{Ca}$ in doped graphene as these metal atoms exhibit rich and better hydrogen binding characteristics than others.

The DFT calculations are performed using the spinpolarized pseudopotential total-energy method as implemented in the Vienna $A b$ initio Simulation Package (VASP). ${ }^{16}$ The projector augmented pseudopotentials are used as provided in the software package. ${ }^{17}$ The electron exchange correlation is treated within both the local-density approximation (LDA) and the generalized gradient approximation (GGA) in the forms of the Ceperley-Alder and the PerdewBurke-Enzelhof type, respectively. ${ }^{18,19}$ The cutoff energy for the plane-wave basis set is chosen to be $400 \mathrm{eV}$, and the atomic relaxation is carried out until the Helmann-Feynman forces of atoms are less than $0.01 \mathrm{eV} / \AA$. To simulate twodimensional (2D) isolated graphenes, a $4 \times 4$ graphene supercell method is used (see Fig. 1). The interlayer distance is set to be $15 \AA$, which is enough to minimize the artificial interlayer interactions. For hydrogen binding, the LDA and GGA are known to provide an upper and a lower bound of a more accurate energy obtained with highly correlated methods such as MP2 or coupled-cluster with singles and doubles and perturbative triples correction $[\operatorname{CCSD}(\mathrm{T})] .^{7,20}$ To investigate the accuracy of the density-functional calculations, part of calculations are repeated using the MP2 in graphene clusters with various basis sets as implemented in NWCHEM packages. $^{21,22}$

Controlling the charge state is very crucial in designing hydrogen storage materials based on metal dispersion. The introduction of acceptor states is a straightforward way to modify the ionic state of metal atoms. For graphenes, substituting boron for carbon is a well-known process in creating the acceptor states. ${ }^{23}$ Figure 1 shows the atomic structure of boron-doped graphenes and the calculated metal binding energy. One, two, or three carbon atoms in carbon hexagon rings are replaced by boron to simulate the boron doping. The binding energy of AEM on these doped graphenes is found to be very sensitive to the boron doping level. Without boron doping, AEMs do not bind to graphene at all $(\mathrm{Mg})$ or will bind very weakly $(\mathrm{Ca})$ as shown in Fig. 1(c). The boron 

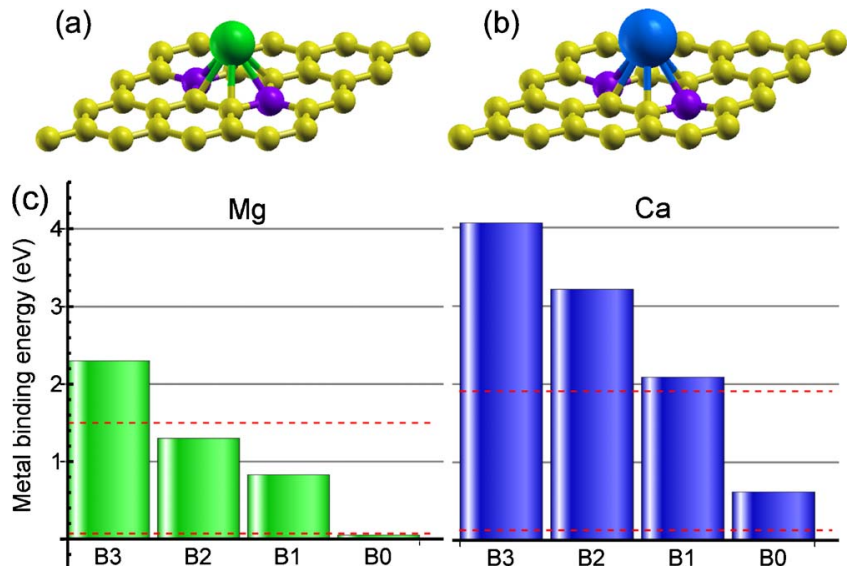

FIG. 1. (Color online) Optimized structure of (a) $\mathrm{Mg}$ and (b) $\mathrm{Ca}$ attached to $\mathrm{B}$-doped graphene $\left(\mathrm{C}_{30} \mathrm{~B}_{2}\right)$. Yellow, purple, large green, and blue balls represent carbon, boron, magnesium, and calcium atoms, respectively. (c) Calculated binding energies of AEM atoms ( $\mathrm{Mg}$ and $\mathrm{Ca}$ ) on $\mathrm{B}$-doped graphenes (each denoted by $\mathrm{B} n$ with $n$ being the number of $\mathrm{B}$ atoms in a carbon hexagon ring without forming B-B bonding). The upper and lower red lines in (c) denote metal bulk and dimer cohesive energies, respectively.

doping, on the other hand, enhances the metal binding significantly, which is crucial in preventing the metal clustering. Our calculated metal binding energy in B-doped graphenes exceeds the metal cohesive energy, which indicates the absence of such clustering. It is also found that segregated two single $\mathrm{Ca}$ atoms in $\mathrm{B}$ defects are energetically more stable than a Ca dimer by about $1 \mathrm{eV}$. Similar trends are also found for TM in the doped graphenes. ${ }^{8,9}$ The acceptorlike states generated by boron change the charge transfer between TM and graphene, leading to a significant improvement in metal dispersion and hydrogen adsorption.

Next, hydrogen adsorption is calculated on AEM atoms dispersed in B-doped graphenes. Among AEMs studied here, $\mathrm{Mg}$ and $\mathrm{Ca}$ exhibit meaningful hydrogen adsorption properties, and results for $\mathrm{Mg}$ - and Ca-graphene complexes are particularly presented here. Only one $\mathrm{H}_{2}$ adsorbs on $\mathrm{Be}$ or $\mathrm{Al}$ complexes at any doping condition considered here according to our LDA calculations. Figure 2 summarized our calculations of the hydrogen adsorption energy at different boron doping levels. The Ca complexes show the best hydrogen adsorption property with respect to the number and binding energy of $\mathrm{H}_{2}$. It is interesting to observe that the orientation of adsorbed hydrogen molecules is not the same for pristine and doped graphenes [see Fig. 2(d)] (its origin will be discussed below). The Mg complexes show a lower hydrogen adsorption capacity than $\mathrm{Ca}$ complexes. Four hydrogen molecules can adsorb at maximum on each $\mathrm{Mg}$ atom in Mg- $\mathrm{C}_{32-x} \mathrm{~B}_{x}$ according to our LDA calculation (it is reduced to three when GGA is used).

It is well-known that the $\mathrm{H}_{2}$ adsorption is not correctly described by either LDA or GGA. ${ }^{7,20,24}$ Detailed comparison with highly correlated methods such as $\operatorname{CCSD}(\mathrm{T})$ is necessary to check the accuracy of the exchange-correlation functionals. As such methods are feasible to very small systems of a few atoms, we chose the MP2 with large basis sets that gives almost the same level of accuracy as $\operatorname{CCSD}(\mathrm{T}) .{ }^{7,20} \mathrm{Hy}$ drogen adsorption energies obtained with DFT are tested in accuracy for $\mathrm{Mg}$ - or $\mathrm{Ca}-\mathrm{C}_{24-x} \mathrm{~B}_{x} \mathrm{H}_{12}$ complex (a cluster form of AEM-graphene complex) using MP2 with available basis sets implemented in NWCHEM. ${ }^{21,22}$ The counterpoise method is employed to correct the basis set superposition errors (BSSE). ${ }^{25,26}$ In MP2 calculations, atomic relaxation was done with $6-31 G^{*}$ basis set. The MP2 binding distance for a single $\mathrm{H}_{2}$ adsorption to $\mathrm{Ca}$ in the coronene is about $2.66 \AA$, which is compared with GGA value of about $2.58 \AA$ (whereas LDA gives the binding distance of about $2.48 \AA$ ). The $\mathrm{H}_{2}$ binding energy to $\mathrm{Mg}$ and $\mathrm{Ca}$ in $\mathrm{C}_{22} \mathrm{~B}_{2} \mathrm{H}_{12}$ was obtained to be about 0.31 and $0.21 \mathrm{eV}$ in MP2 calculations with correlation consistent polarized valence only triple-zeta (ccpVTZ) and $6-311++\mathrm{G}(2 d, 2 p)$ basis sets for $\mathrm{Mg}$ and $\mathrm{Ca}$, respectively. The GGA calculation gives about $10 \%$ smaller values than MP2, whereas LDA values are almost two times larger. The $\mathrm{Ca}-\mathrm{H}_{2}$ binding distance and $\mathrm{H}-\mathrm{H}$ bond length on average are 2.64 and $0.76 \AA$, and 2.43 and $0.78 \AA$ for
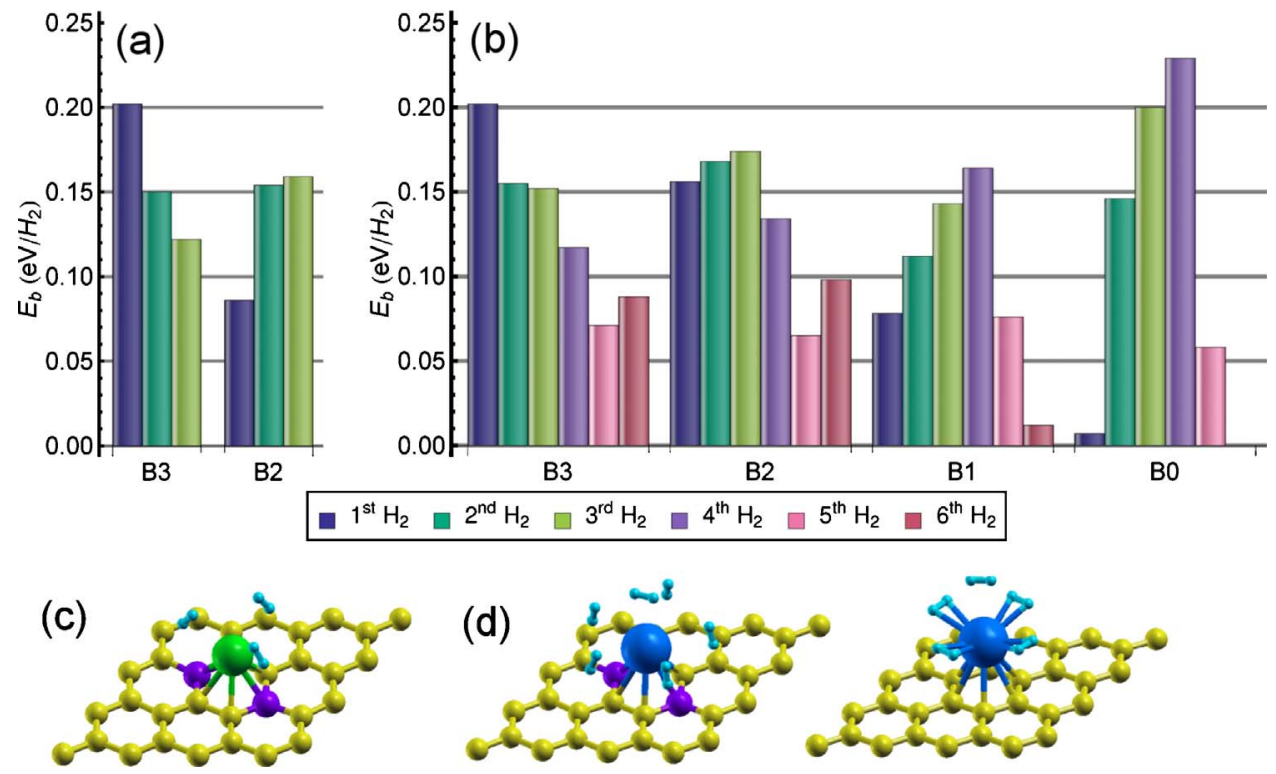

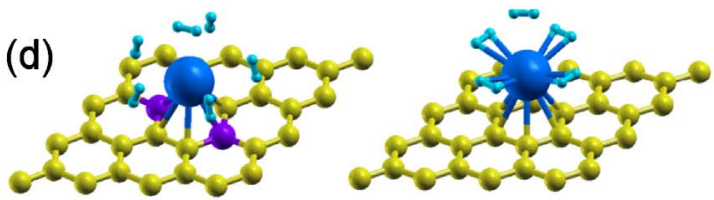

FIG. 2. (Color online) Consecutive $\mathrm{H}_{2}$ binding energies to (a) Mg- and (b) $\mathrm{Ca}-\mathrm{C}_{32-x} \mathrm{~B}_{x}$ complexes in GGA. The label in the abscissa is the same as in Fig. 1(c). The optimized structures of (c) $3 \mathrm{H}_{2}+\mathrm{Mg}-\mathrm{C}_{30} \mathrm{~B}_{2}$ and (d) 6 (or 5) $\mathrm{H}_{2}+\mathrm{Ca}-\mathrm{C}_{32-x} \mathrm{~B}_{x}$ are also shown below. 

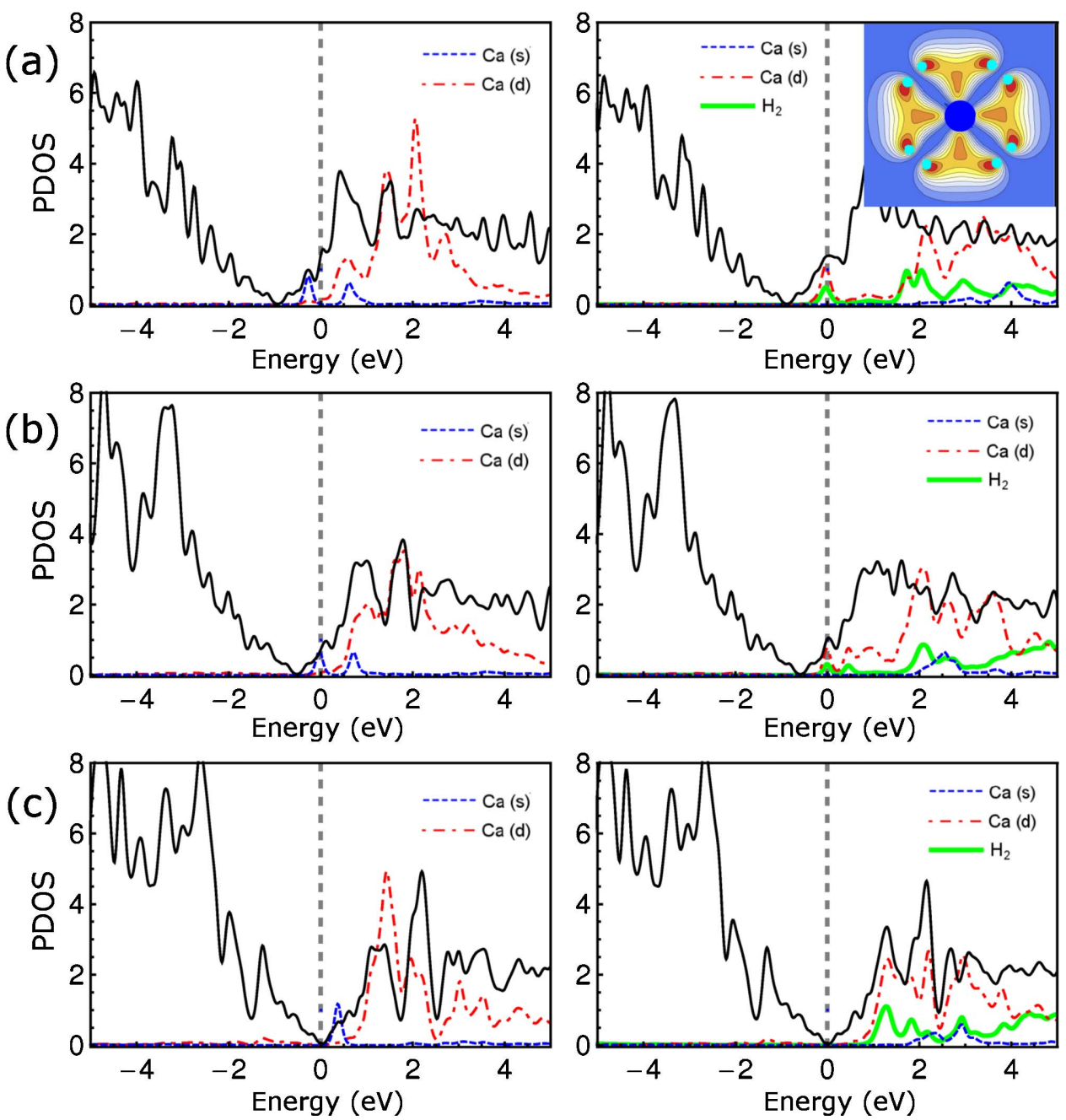

FIG. 3. (Color online) Calculated partial density of states (PDOS) of $\mathrm{Ca}-\mathrm{C}_{32-x} \mathrm{~B}_{x}$ for $x=(\mathrm{a})$ 0 , (b) 1 , and (c) 2 in units of state/eV cell. The Ca $s$ states here are enlarged twofold for a clear view. The left and right panels indicate PDOS for without $\mathrm{H}_{2}$ and with $\mathrm{H}_{2}$ saturated, respectively. The inset of (a) shows the planar charge distribution of the states just below the Fermi level in the $\mathrm{Ca}-4 \mathrm{H}_{2}$ plane, where the contour interval is $0.004 e / \AA^{3}$.
$6 \mathrm{H}_{2}+\mathrm{Ca}-\mathrm{C}_{30} \mathrm{~B}_{2}$ and $5 \mathrm{H}_{2}+\mathrm{Ca}-\mathrm{C}_{32}$, respectively (the bond length of isolated $\mathrm{H}_{2}$ is calculated to be about $0.75 \AA$ with GGA). Since $\mathrm{H}_{2}$ physisorption on graphitic materials is not reproduced at all in GGA, ${ }^{7,20,24}$ this observation implies that $\mathrm{H}_{2}$ adsorption on AEM is not in the physisorption regime.

Our calculations show that each $\mathrm{Ca}$ atom can hold up to six $\mathrm{H}_{2}$ (both in LDA and GGA), which is one $\mathrm{H}_{2}$ more than in previous reports ${ }^{27}$ and for TM atoms. ${ }^{5-10}$ The calculated $\mathrm{H}_{2}$ adsorption energy in AEM- $\mathrm{C}_{32-x} \mathrm{~B}_{x}$ complexes is, on the other hand, smaller than that in TM-graphene complexes (for example, by about $50 \%$ on average for Sc complexes ${ }^{6,9,10}$ ). The $\mathrm{H}-\mathrm{H}$ bond length $\left(\mathrm{d}_{\mathrm{H}-\mathrm{H}}\right)$ and the metal- $\mathrm{H}_{2}$ binding distance $\left(\mathrm{d}_{\mathrm{M}-\mathrm{H} 2}\right)$ consistently indicate a weaker binding of hydrogen in AEM- $\mathrm{C}_{32-x} \mathrm{~B}_{x}$ complexes. The desorption temperature of $\mathrm{H}_{2}$ will accordingly be lower than that for TM complexes but it is still in an acceptable range for practical application. . $^{3,7,24}$ The hydrogen adsorption on $\mathrm{Ca}$ exhibits intriguing features. The adsorption energy of the first (the fourth) hydrogen molecule increases (decreases) as boron concentration increases. The order in binding energy of the first four $\mathrm{H}_{2}$ also changes as boron concentration increases. Without boron doping, the $\mathrm{H}_{2}$ adsorbed later has a larger adsorption energy whereas it is opposite in $\mathrm{C}_{29} \mathrm{~B}_{3}$. Another interesting observation is the orientation of $\mathrm{H}_{2}$. As Fig. 2(d) demonstrates, the orientation of $\mathrm{H}_{2}$ relative to graphene plane makes a drastic change upon boron doping. Without boron doping, $\mathrm{H}-\mathrm{H}$ bond lies parallel to graphene plane. However, it turns almost vertical to the plane for $\mathrm{C}_{30} \mathrm{~B}_{2}$. All these features can be explained by a crossover in $\mathrm{Ca}-\mathrm{H}_{2}$ interactions from Kubas to multipole Coulomb interactions. The energy level and occupation of $s$ and $d$ orbitals of $\mathrm{Ca}$ is significantly affected by hydrogen adsorption and boron doping, and so is the interaction between $\mathrm{Ca}$ and $\mathrm{H}_{2}$.

Of particular interest is the role of the empty $d$ orbitals of $\mathrm{Ca}$ for hydrogen adsorption on Ca complexes. In TM dispersion, it was shown that partially filled $d$ orbitals strengthen the $\mathrm{H}_{2}$ binding to TM but reduces the number of bound $\mathrm{H}_{2} \cdot{ }^{8,9}$ In order to investigate the role of low-lying Ca $d$ orbitals, the electronic structure of $\mathrm{Ca}$ complexes is studied with or without boron doping and also upon successive hydrogen adsorption. Figure 3 shows the calculated density of states (DOS) projected onto atomic orbitals. When $\mathrm{Ca}$ atoms bind to pristine graphenes, one $s$ electron of $\mathrm{Ca}$ transfers to graphene. The initial hydrogen adsorption on $\mathrm{Ca}$ atoms in this case is mostly mediated by the dipole interaction between $\mathrm{Ca}^{+}$ion and $\mathrm{H}_{2}$ (induced) dipole moment. As more hydrogen molecules are adsorbed on $\mathrm{Ca}$, the repulsive potential of hydrogen electrons pushes $\mathrm{Ca} s$ orbital into higher energy than that of $\mathrm{Ca} d$ orbitals, which then become partially occupied. When this $s$ - $d$ level exchange occurs, Kubas interaction be- 
comes dominant providing stronger and a more stable configuration. This explains the small adsorption energy of the first hydrogen molecule and the increasing order in the adsorption energy of $\mathrm{H}_{2}$ on $\mathrm{Ca}$ in pristine graphene as shown in Fig. 2(b). The inset of Fig. 3(a) clearly shows the bonding characteristics of Kubas interactions between $\mathrm{H}_{2}$ and $\mathrm{Ca}$ with $\mathrm{H}_{2}$ bond aligned parallel to graphene plane (such charge distribution is not observed when only the static Coulomb interaction involves in $\mathrm{H}_{2}$ binding, for example, at boron doublet and triplet defects).

When boron replaces carbon, the crossover occurs in the other way. Boron creates localized and partially occupied states near the Fermi level at low boron concentrations. Once metal $(\mathrm{Mg}$ or $\mathrm{Ca})$ atoms bind to the defect sites, the boronderived states are filled by the electrons from metal $s$ orbital. This leads to a stronger metal binding to the substrates, ${ }^{8,9}$ which, in turn, prevents metal clustering. The trend in charge transfer from Ca $s$ orbital to boron-induced acceptor states changes drastically as $\mathrm{H}_{2}$ adsorbs on Ca. Without hydrogen adsorption, the relative position in energy level of graphene, and $\mathrm{Ca} s$ and $d$ states remains almost the same except for Fermi-level shift as boron concentration increases. When hydrogen adsorbs on $\mathrm{Ca}$, on the other hand, a small peak from Ca $d$ level and the hydrogen $\sigma^{*}$ states are partially occupied while the main peak from $\mathrm{Ca} d$ level shifts down in energy. Moreover the Ca $s$ states also rise in energy becoming unoccupied as hydrogen $s$ electrons are repulsive to $\mathrm{Ca} s$ electrons as mentioned above. On the other hand, the small peak of $\mathrm{Ca} d$ character disappears and hydrogen $\sigma^{*}$ states become empty at larger boron concentrations \{double [Fig. 3(c)] and triple (not shown here) boron substitutions\}. The ionic state of $\mathrm{Ca}$ atoms also changes from +1 to +2 . The Kubas interaction, which is mediated by electron backdonation to hydrogen $\sigma^{*}$ states, thus becomes less effective at this boron concentration. The multipole Coulomb interaction between $\mathrm{Ca}^{2+}$ ion, localized charges near boron defects, and hydrogen dipole and quadruple moments come into play. These features are also confirmed by the MP2 calculations for $\mathrm{Ca}^{1+}$ and $\mathrm{Ca}^{2+}$ ions with hydrogen adsorbed. We note that the side-on adsorption of $\mathrm{H}_{2}$ to $\mathrm{Ca}^{2+}$ can be well described by the interaction between the metal ion and $\mathrm{H}_{2}$ quadruple moments as discussed in literatures. ${ }^{14,28}$

The adsorption energy in this regime is thus the most significant for the first hydrogen molecule which is consistent with the results in Fig. 2. Calculated DOS and the $\mathrm{H}_{2}$ orientation imply that the $\mathrm{H}_{2}$ adsorption making a crossover from Kubas to multipole Coulomb interaction occurs earlier as boron concentration increases. For TM, the best metal dispersion and hydrogen adsorption were obtained at large boron concentrations (triple boron substitution), which was found to be energetically unfavorable, if not serious. ${ }^{9}$ For AEM, the crossover in the interaction between $\mathrm{Ca}$ and $\mathrm{H}_{2}$, on the other hand, makes the optimal hydrogen adsorption be realized at smaller boron concentrations.

One practical issue is how to maintain pores large enough for both $\mathrm{Ca}$ dispersion and hydrogen adsorption in graphenemetal complexes. Intercalation of $\mathrm{Ca}$ atoms into graphite separates the graphene layers by about $4.5 \AA$ but this is not enough for having maximal $\mathrm{H}_{2}$ molecules. Also a direct use of graphenes for hydrogen storage may not be useful as they

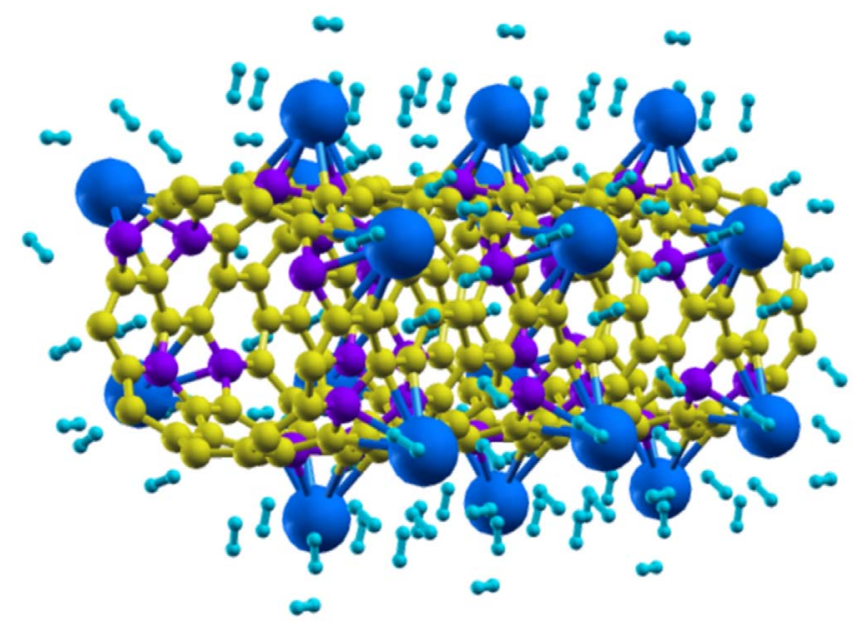

FIG. 4. (Color online) Optimized geometries of hydrogen molecules maximally adsorbed on the Ca-decorated B-doped $(9,0)$ zigzag carbon nanotube whose chemical formation is $\mathrm{C}_{60} \mathrm{~B}_{12}\left[\mathrm{Ca}\left(\mathrm{H}_{2}\right)_{6}\right]_{6}$. Here the storage capacity is about $6.2 \mathrm{wt} \%$ with the average $\mathrm{H}_{2}$ binding energy of about $0.13 \mathrm{eV} / \mathrm{H}_{2}$.

might cluster. Porous three-dimensional (3D) structures based on graphenes should be utilized as meaningful hydrogen storage. The task of building 3D structures that have large pores with structural stability is a real challenge. For example, organic frameworks have been pursued extensively but the chemical constraint in their structures limits the incorporation of various metal atoms with desirable coordination number. Separation of graphite layers by intercalation of large molecules or atoms such as $\mathrm{C}_{60}$ (Ref. 29) was proposed but the stability and the metal dispersion are still questionable. A computational model of purely graphitic 3D structure was also proposed, namely, covalently bonded graphene $(\mathrm{CBG}),{ }^{7,30}$ which can be constructed by linking edge atoms of graphene stripes with $s p^{3}$ bonds. As the CBG structure was shown to be very stable ${ }^{30}$ and also be effective in metal dispersion at its $s p^{3}$-bonded edge (vertex) sites, ${ }^{7}$ they may also be good candidates. Recently Dimitrakakis et al. ${ }^{31}$ introduced 3D graphene structure whose interlayer separation is sustained by carbon nanotubes (CNTs) which are covalently bonded to pores in graphenes.

As such 3D templates are applicable to our study dealing with B-doped graphenes and CNTs, we also tested B-doped CNTs for $\mathrm{Ca}$ and successive $\mathrm{H}_{2}$ bindings. With experimentally available B concentration of about 25 at. \% in graphitic carbons, ${ }^{32}$ the storage capacity can also be estimated from calculated hydrogen adsorption on $\mathrm{Ca}$ (for example, CNT, activated carbon, or graphene fragments doped with B can be used as substrates to disperse $\mathrm{Ca}$ atoms). Figure 4 shows the atomic structure of the Ca-dispersed B-doped CNT with multiple $\mathrm{H}_{2}$ adsorbed on $\mathrm{Ca}$ atoms. The boron concentration here is about 20 at. \%, and the storage capacity is estimated to be about 6.2 wt \%. Previous reports on Ca-dispersed fullerenes showed similar results. ${ }^{27}$ As their stability against clustering is provided by such boron defect sites (and also with conception of CNT pillars between graphene layers ${ }^{31}$ ), activated carbon of high surface area with boron and $\mathrm{Ca}$ dopings can be good 3D hydrogen storage materials. Again we note that 
building 3D structures for effective hydrogen storage systems is still an ongoing issue that must incorporate a number of possibilities and trials to be solved.

In summary, boron-doped graphenes were found to be effective for dispersion of AEM, particularly $\mathrm{Ca}$, and hydrogen adsorption thereon, which is advantageous for hydrogen storage. The $\mathrm{H}_{2}$ adsorption in such complexes exhibits a crossover between Kubas and multipole Coulomb interactions depending on the ionic state of AEM and the number of adsorbed $\mathrm{H}_{2}$. Our study suggests that material characteristics such as doping conditions can be optimized utilizing such an understanding of the adsorption mechanism in AEM- dispersed materials. Based on our results, 3D structures with CNT pillars between graphene layers is also suggested as good templates for $\mathrm{Ca}$ dispersion and hydrogen storage systems.

This research was performed for the Hydrogen Energy R\&D Center, one of the 21st Century Frontier R\&D Program, funded by the Ministry of Science and Technology of Korea. This work was also supported by Grant No. KSC2007-S00-2004 from Korea Institute of Science and Technology Information.
*Corresponding author; jhish@ postech.ac.kr

${ }^{1}$ L. Schlapbach and A. Züttel, Nature (London) 414, 353 (2001).

${ }^{2}$ M. Fichtner, Adv. Eng. Mater. 7, 443 (2005).

${ }^{3}$ S.-H. Jhi and Y.-K. Kwon, Phys. Rev. B 69, 245407 (2004).

${ }^{4}$ S.-H. Jhi, Phys. Rev. B 74, 155424 (2006).

${ }^{5}$ T. Yildirim and S. Ciraci, Phys. Rev. Lett. 94, 175501 (2005).

${ }^{6}$ H. Lee, W. I. Choi, and J. Ihm, Phys. Rev. Lett. 97, 056104 (2006).

${ }^{7}$ N. Park, S. Hong, G. Kim, and S.-H. Jhi, J. Am. Chem. Soc. 129, 8999 (2007).

${ }^{8}$ G. Kim, N. Park, and S.-H. Jhi, Appl. Phys. Lett. 92, 013106 (2008).

${ }^{9}$ G. Kim, S.-H. Jhi, N. Park, S. G. Louie, and M. L. Cohen, Phys. Rev. B 78, 085408 (2008).

${ }^{10}$ Y. Zhao, Y.-H. Kim, A. C. Dillon, M. J. Heben, and S. B. Zhang, Phys. Rev. Lett. 94, 155504 (2005).

${ }^{11}$ G. J. Kubas, J. Organomet. Chem. 635, 37 (2001).

${ }^{12}$ Q. Sun, Q. Wang, P. Jena, and Y. Kawazoe, J. Am. Chem. Soc. 127, 14582 (2005).

${ }^{13}$ S. Li and P. Jena, Phys. Rev. Lett. 97, 209601 (2006).

${ }^{14}$ R. C. Lochan and M. Head-Gordon, Phys. Chem. Chem. Phys. 8, 1357 (2006).

${ }^{15}$ Chr. Møller and M. S. Plesset, Phys. Rev. 46, 618 (1934).

${ }^{16}$ G. Kresse and J. Furthmüller, Phys. Rev. B 54, 11169 (1996).

${ }^{17}$ G. Kresse and D. Joubert, Phys. Rev. B 59, 1758 (1999).
${ }^{18}$ D. M. Ceperley and B. J. Alder, Phys. Rev. Lett. 45, 566 (1980).

${ }^{19}$ J. P. Perdew, K. Burke, and M. Ernzerhof, Phys. Rev. Lett. 77, 3865 (1996).

${ }^{20}$ A. Ferre-Vilaplana, J. Chem. Phys. 122, 104709 (2005).

${ }^{21}$ E. J. Bylaska et al., Pacific Northwest National Laboratory, Richland, Washington 99352-0999, 2006.

${ }^{22}$ R. A. Kendall et al., Comput. Phys. Commun. 128, 260 (2000).

${ }^{23}$ D. L. Carroll, Ph. Redlich, X. Blase, J.-C. Charlier, S. Curran, P. M. Ajayan, S. Roth, and M. Ruhle, Phys. Rev. Lett. 81, 2332 (1998).

${ }^{24}$ Y.-H. Kim, Y. Zhao, A. Williamson, M. J. Heben, and S. B. Zhang, Phys. Rev. Lett. 96, 016102 (2006).

${ }^{25}$ H. B. Jansen and P. Ros, Chem. Phys. Lett. 3, 140 (1969).

${ }^{26}$ S. B. Boys and F. Bernardi, Mol. Phys. 100, 65 (2002).

${ }^{27}$ M. Yoon, S. Yang, C. Hicke, E. Wang, D. Geohegan, and Z. Zhang, Phys. Rev. Lett. 100, 206806 (2008).

${ }^{28}$ M. Yoon, S. Yang, E. Wang, and Z. Zhang, Nano Lett. 7, 2578 (2007).

${ }^{29}$ V. Gupta, P. Scharff, K. Rischa, H. Romanus, and R. Müller, Solid State Commun. 131, 153 (2004).

${ }^{30}$ N. Park and J. Ihm, Phys. Rev. B 62, 7614 (2000).

${ }^{31}$ G. K. Dimitrakakis, E. Tylianakis, and G. E. Froudakis, Nano Lett. 8, 3166 (2008).

${ }^{32}$ Y. Ferro, F. Marinelli, and A. Allouche, J. Chem. Phys. 118, 5650 (2003). 